Monika Lindner, Center of Mathematics and Physics, Technical University of Łódź, Al. Politechniki 11 90-924 Łódź, Poland. email:

mlindner@p.lodz.pl

Sebastian Lindner, Faculty of Mathematics and Computer Science, University of Łódź, 90-238 Łódź, Banacha 22, Poland. email:

lindner@math.uni.lodz.pl

\title{
CHARACTERIZATIONS OF SOME SUBCLASSES OF THE FIRST CLASS OF BAIRE
}

\begin{abstract}
In the paper [3] the authors have examined functions of the Baire class 1 , where the domain and the range were metric spaces. The $\varepsilon-\delta$ characterization of such functions has been proved. In this note we examine, if replacing of the condition from [3] by it's stronger version can lead us to the characterization of some subclass of $B_{1}$ on the interval $[0,1]$.
\end{abstract}

\section{Definition of the class $B_{A}$. Basic properties}

In 2000 year Lee, Tang and Zhao published the following theorem:

Theorem ([3]) Suppose that $f: X \rightarrow \mathbf{R}$ is a real valued function on a complete separable metric space $X$. Then the following statements are equivalent:

(1) For every $\varepsilon>0$ there exists a positive function $\delta$ on $X$ such that

$$
\left|f\left(x_{1}\right)-f\left(x_{2}\right)\right|<\varepsilon
$$

whenever

$$
d_{X}\left(x_{1}, x_{2}\right)<\min \left(\delta\left(x_{1}\right), \delta\left(x_{2}\right)\right) .
$$

Mathematical Reviews subject classification: Primary: 26A21

Key words: Baire one class, approximate continuity, Darbou'x continuity

Received by the editors February 17, 2011

Communicated by: Miroslav Zelený 
(2) The function $f$ is of Baire class one.

Following Atok, Tang and Zhao ([4]) we call the positive function $\delta$ in (1) an $\varepsilon$-gauge of $f$.

We restrict our considerations to the case of real functions defined on $[0,1]$ interval. The question is: If we use only gauges $\delta$ meeting some extra conditions, are we supposed to obtain the class smaller then $B_{1}$ ? The answer is positive. To express it more precisely consider the following definition :

Definition 1 Let $A$ be an arbitrary family of real valued functions defined on the interval $[0,1], f:[0,1] \rightarrow \mathbf{R}$. We say that $f \in B_{A}$, iff for every $\varepsilon>0$ there exists $\delta:[0,1] \rightarrow(0, \infty)$ such that $\delta \in A$ and for every $x_{1}, x_{2} \in[0,1]$ the following implication holds

$$
\left|x_{1}-x_{2}\right|<\min \left(\delta\left(x_{1}\right), \delta\left(x_{2}\right)\right) \quad \Longrightarrow \quad\left|f\left(x_{1}\right)-f\left(x_{2}\right)\right|<\varepsilon .
$$

In the sequel let $B 1, C$, lsc, usc, $D$, app, ls-app, $B 1^{*}$ denote respectively classes of Baire 1, continuous, lower semicontinuous, upper semicontinuous, Darboux continuous, approximately continuous, lower semi approximately continuous functions and Baire* one functions defined on $[0,1]$.

(Function $f:[0,1] \rightarrow \mathbf{R}$ is lower semi approximately continuous iff for each $a \in \mathbf{R}$ the set $f^{-1}((a, \infty))$ is open with respect to the density topology on $[0,1]$.

Function $f:[0,1] \rightarrow \mathbf{R}$ belongs to $B 1^{*}$ iff for every closed set $F \subset[0,1]$ there exists the interval $(a, b)$ such that $(a, b) \cap F \neq \emptyset$ and the function $f$ restricted to $(a, b) \cap F$ is continuous.)

Proposition 1 The operator $A \rightarrow B_{A}$ has the following properties:

1. If $A_{1} \subset A_{2} \subset \mathbf{R}^{[0,1]}$ then $B_{A_{1}} \subset B_{A_{2}} \subset B 1$,

2. For every family $A \subset \mathbf{R}^{[0,1]}$ the family $B_{A}$ is closed under uniform convergence,

3. If the family $A \subset \mathbf{R}^{[0,1]}$ satisfies the following condition:

$$
\min (f, g) \in A \text { for every } f, g \in A,
$$

then the family $B_{A}$ forms a linear subspace of the space $\mathbf{R}^{[0,1]}$. 
Proof.

1. The first inclusion follows directly from the definition of the family $B_{A}$. The second inclusion follows from the Lee, Tang and Zao theorem.

2. Let $f_{n} \in B_{A}$ for $n \in \mathbf{N}$, and the sequence $\left(f_{n}\right)$ converges uniformly to the function $f$. Take $\varepsilon>0$. Let $n \in \mathbf{N}$ be large enough to get

$$
\left|f(x)-f_{n}(x)\right|<\frac{\varepsilon}{3}
$$

for every $x \in[0,1]$. Let $\delta \in A$ be an $\frac{\varepsilon}{3}$-gauge of the function $f_{n}$. Take arbitrary $a, b \in[0,1]$ such that $|a-b|<\min (\delta(a), \delta(b))$. Then

$$
|f(a)-f(b)| \leq\left|f(a)-f_{n}(a)\right|+\left|f_{n}(a)-f_{n}(b)\right|+\left|f_{n}(b)-f(b)\right|<\varepsilon .
$$

So the function $\delta$ is an $\varepsilon$-gauge of $f$. Hence $f \in B_{A}$.

3. Let $f, g \in B_{A}$, let $\alpha \in \mathbf{R}, \varepsilon>0$. Let $\delta \in A$ be an $\left(\varepsilon \cdot(\max (|\alpha|, 1))^{-1}\right)$-gauge of $f$. Then $\delta$ is an $\varepsilon$-gauge of $\alpha f$. Let $\delta_{1}$ be the $\left(\frac{\varepsilon}{2}\right)$-gauge of $f$ and $\delta_{2}$ be the $\left(\frac{\varepsilon}{2}\right)$-gauge of $g$. Then the function $\min \left(\delta_{1}, \delta_{2}\right)$ is an $\varepsilon$-gauge of $f+g$.

\section{Results}

Theorem $1 B_{\text {const }}=B_{C}=B_{l s c}=C$.

Proof. We have $B_{\text {const }} \subset B_{C} \subset B_{\text {lsc }}$ because const $\subset C \subset$ lsc.

Let $f \in B_{\mathrm{lsc}}, \varepsilon>0$. Let $\delta \in$ lsc be an $\varepsilon$-gauge of $f$. As the function $\delta$ is positive and lower semicontinuous, it has the positive lower bound $\eta$ on $[0,1]$. Hence, for every $a, b \in[0,1]$, the condition $|a-b|<\eta$ implies $|f(a)-f(b)|<\varepsilon$. Therefore the function $f$ is uniformly continuous on $[0,1]$.

Let $f \in C$. Then $f$ is uniformly continuous. For every $\varepsilon>0$ there exists $\eta>0$ such that $|f(a)-f(b)|<\varepsilon$ if $|a-b|<\eta$. Defining $\delta(x)=\eta$ for $x \in[0,1]$, we obtain a constatnt $\varepsilon$-gauge of $f$.

Let $x_{0}$ be a right hand side accumulation point of the set $E \subset \mathbf{R}$. By $\operatorname{Lim}_{x \rightarrow x_{0}+} f(x)$ we shall denote the set of all limits of sequences of the form $f\left(x_{n}\right)$, where $\left(x_{n}\right)$ is the sequence of points belonging to $E \cap\left(x_{0}, \infty\right)$ converging to $x_{0}$. The set $\operatorname{Lim}_{x \rightarrow x_{0}+} f(x)$ is always closed. The definition of $\operatorname{Lim}_{x \rightarrow x_{0}-} f(x)$ is analogous. 
Let us recall the characterization of Darboux continuous functions among the Baire one functions:

Theorem ([1], chap. 2, th. 1.1) Let $f:[0,1] \rightarrow \mathbf{R}$ be of Baire class one. Then the following conditions are equivalent

(1) $f$ has the Darboux property;

(2) $f\left(x_{0}\right) \in \operatorname{Lim}_{x \rightarrow x_{0}-} f(x) \cap \operatorname{Lim}_{x \rightarrow x_{0}+} f(x)$ for every $x_{0} \in[0,1]$.

(In case when $x_{0} \in\{0,1\}$ the second condition has its unilateral version.)

Theorem $2 B_{D} \subset D$.

Proof. Let $f \in B_{D}$. Of course $f \in B_{1}$. Suppose that $f \notin D$. From the last theorem we have existence of $x_{0} \in[0,1]$, such that $f\left(x_{0}\right) \notin \operatorname{Lim}_{x \rightarrow x_{0}-} f(x) \cap$ $\operatorname{Lim}_{x \rightarrow x_{0}+} f(x)$ For instance let the distance between $f\left(x_{0}\right)$ and $\operatorname{Lim}_{x \rightarrow x_{0}-} f(x)$ equals $2 \varepsilon$ for some positive number $\varepsilon$. Let $\delta$ be an $\varepsilon$-gauge of $f$. We shall show, that $\delta$ cannot be Darboux continuous. Let $\left(x_{n}\right)_{n \in \mathbf{N}}$ be an arbitrary choosen sequence of points belonging to $\left[0, x_{0}\right)$ converging to $x_{0}$. There exists $N_{1}$ such that $\left|x_{n}-x_{0}\right|<\delta\left(x_{0}\right)$ for $n>N_{1}$. And there exists $N_{2}$ such that $\left|f\left(x_{n}\right)-f\left(x_{0}\right)\right|>\varepsilon$ for $n>N_{2}$.

Therefore for $n>\max \left(N_{1}, N_{2}\right)$ we have $\left|x_{n}-x_{0}\right| \geq \delta\left(x_{n}\right)$. Hence $\lim _{x \rightarrow x_{0}-} \delta(x)=$ 0 . But $\delta\left(x_{0}\right)>0$, so $\delta$ is not Darboux continuous.

Problem 1 Does the opposite inclusion hold: $B 1 \cap D \subset B_{D}$ ?

Theorem $3 B_{l s-a p p} \subset$ app.

Proof. Let $f \in B_{\mathrm{ls}-\mathrm{app}}, \varepsilon>0, x_{0} \in[0,1]$. We shall demonstrate, that $x_{0}$ is a density point of the set $Z\left(x_{0}, \varepsilon\right)=f^{-1}\left(\left(f\left(x_{0}\right)-\varepsilon, f\left(x_{0}\right)+\varepsilon\right)\right)$. Let $\delta$ be an approximately lower semicontinuous $\varepsilon$-gauge of $f$. Then $x_{0}$ is the density point of the set

$$
T\left(x_{0}\right)=\delta^{(-1)}\left(\left(\frac{\delta\left(x_{0}\right)}{2}, \infty\right)\right)
$$

Let $x$ be an arbitrary point of $T\left(x_{0}\right)$ such that $\left|x-x_{0}\right|<\frac{\delta\left(x_{0}\right)}{2}$. Then $\left|x-x_{0}\right|<$ $\min \left(\delta(x), \delta\left(x_{0}\right)\right)$, so $\left|f(x)-f\left(x_{0}\right)\right|<\varepsilon$. Hence $x \in Z\left(x_{0}, \varepsilon\right)$. Therefore

$$
T\left(x_{0}\right) \cap\left(x_{0}-\frac{\delta\left(x_{0}\right)}{2}, x_{0}+\frac{\delta\left(x_{0}\right)}{2}\right) \subset Z\left(x_{0}, \varepsilon\right) .
$$


But $x_{0}$ is a density point of $T\left(x_{0}\right) \cap\left(x_{0}-\frac{\delta\left(x_{0}\right)}{2}, x_{0}+\frac{\delta\left(x_{0}\right)}{2}\right)$, hence it is also the density point of $Z\left(x_{0}, \varepsilon\right)$.

Let us recall the notion of oscillation index of function. Let $f:[0,1] \rightarrow \mathbf{R}$ and $\varepsilon>0$. For each $A \subset[0,1]$ let

$$
P_{\varepsilon, f}(A)=\{x \in A: \operatorname{osc}(f, x, A) \geq \varepsilon\} .
$$

Let us define the transfinite sequence of sets $\left(F_{f, \varepsilon}^{\alpha}\right)_{\alpha<\omega_{1}}$ in the following way:

$$
F_{f, \varepsilon}^{\alpha}= \begin{cases}{[0,1]} & \text { for } \alpha=0 \\ P_{\varepsilon, f}\left(F_{f, \varepsilon}^{\gamma}\right) & \text { for } \alpha=\gamma+1 \\ \bigcap_{\gamma<\alpha} F_{f, \varepsilon}^{\gamma} & \text { if } \alpha \text { is a limit ordinal. }\end{cases}
$$

If there exist $\alpha<\omega_{1}$ such that $F_{f, \varepsilon}^{\alpha}=\emptyset$ then let $\beta(f, \varepsilon)=\min \left\{\alpha: F_{f, \varepsilon}^{\alpha}=\emptyset\right\}$. In case that for every $\alpha<\omega_{1} F_{f, \varepsilon}^{\alpha} \neq \emptyset$ let $\beta(f, \varepsilon)=\omega_{1}$. Finally let $\beta(f)=$ $\sup _{\varepsilon>0} \beta(f, \varepsilon)$.

Let us recall the following

Theorem ([2]) Let $f:[0,1] \rightarrow \mathbf{R}$. Then

(1) $\beta(f)=1$ iff $f$ is continuous,

(2) $\beta(f)<\omega_{1}$ iff $f \in B 1$.

The next theorem shows the connection between the oscillation index of the function $f$ and the oscillation index of its gauge:

Theorem 4 Let $f:[0,1] \rightarrow \mathbf{R}, \beta(f)=\alpha<\omega_{1}$. Then for every $\varepsilon>0$ there exists an $\varepsilon$-gauge $\delta$ of $f$ such that $\delta \in$ usc $\cap B 1^{*}$ and $\beta(\delta) \leq \alpha$.

Proof. Let $\varepsilon>0$. We shall construct a gauge $\delta$ using the sequence $\left\{F_{f, \varepsilon}^{\gamma}\right\}_{\gamma \leq \alpha}$ defined in the definition of the oscillation index. Notice that

$$
[0,1]=\bigcup_{\gamma<\alpha}\left(F_{f, \varepsilon}^{\gamma} \backslash F_{f, \varepsilon}^{\gamma+1}\right)
$$

Suppose that $\gamma<\alpha$ and the value $\delta(x)$ has been already defined for $x \in$ $\bigcup_{\xi<\gamma}\left(F_{f, \varepsilon}^{\xi} \backslash F_{f, \varepsilon}^{\xi+1}\right)=[0,1] \backslash F_{f, \varepsilon}^{\gamma}$. Now we define the function $\delta$ on the set $F_{f, \varepsilon}^{\gamma} \backslash F_{f, \varepsilon}^{\gamma+1}$. 
Let $t \in F_{f, \varepsilon}^{\gamma} \backslash F_{f, \varepsilon}^{\gamma+1}$. According to the definition of the sequence $\left\{F_{f, \varepsilon}^{\gamma}\right\}_{\gamma \leq \alpha}$ the oscillation of the function $f$ restricted to the set $F_{f, \varepsilon}^{\gamma}$ at the point $t$ is less than $\varepsilon$. So there exists an open neighbourhood $V_{t}$ of $t$, such that $\operatorname{diam}\left(f\left(V_{t} \cap\right.\right.$ $\left.\left.F_{f, \varepsilon}^{\gamma}\right)\right)<\varepsilon$. Let us define the function $\delta^{t}(x)=\frac{1}{2} d\left(x,[0,1] \backslash V_{t}\right)$, where $d(x, A)$ stands for the distance between the point $x$ and the set $A$. The function $\delta^{t}$ satisfies the Lipschitz condition with the constant 1.

Let

$$
\delta(x)=\sup _{t \in F_{f, \varepsilon}^{\gamma} \backslash F_{f, \varepsilon}^{\gamma+1}} \delta^{t}(x)
$$

for $x \in F_{f, \varepsilon}^{\gamma} \backslash F_{f, \varepsilon}^{\gamma+1}$. Observe that the function $\delta$ is strictly positive. It is also continuous on the set $F_{f, \varepsilon}^{\gamma} \backslash F_{f, \varepsilon}^{\gamma+1}$ as an upper bound of an equi-continuous family of functions.

In this way we have defined the gauge $\delta$ on the whole interval $[0,1]$.

Now we shall examine the properties of $\delta$.

(1) Let $x \in[0,1], x \in F_{f, \varepsilon}^{\gamma} \backslash F_{f, \varepsilon}^{\gamma+1}, \gamma<\alpha$. Then $(x-\delta(x), x+\delta(x)) \cap F_{f, \varepsilon}^{\gamma+1}=\emptyset$. In fact, by the definition of $\delta$, there exists $t \in F_{f, \varepsilon}^{\gamma} \backslash F_{f, \varepsilon}^{\gamma+1}$, such that $\delta^{t}(x)>$ $\frac{3}{4} \delta(x)$. Therefore there exists a set $V_{t}$ such that $\operatorname{diam}\left(f\left(V_{t} \cap F_{f, \varepsilon}^{\gamma}\right)\right)<\varepsilon$ and $d\left(x,[0,1] \backslash V_{t}\right)=2 \delta^{t}(x)>\frac{3}{2} \delta(x)$. Hence $(x-\delta(x), x+\delta(x)) \subset V_{t}$ and $V_{t} \cap$ $F_{f, \varepsilon}^{\gamma+1}=\emptyset$.

(2) If two points $x, y \in[0,1]$ fullfill the condition $|x-y|<\min (\delta(x), \delta(y))$ then for some ordinal $\gamma$ we have $x, y \in F_{f, \varepsilon}^{\gamma} \backslash F_{f, \varepsilon}^{\gamma+1}$ and $|f(x)-f(y)|<\varepsilon$. Hence $\delta$ is an $\varepsilon$-gauge of $f$.

Proof. Let $x \in F_{f, \varepsilon}^{\gamma} \backslash F_{f, \varepsilon}^{\gamma+1}$ and $y \in F_{f, \varepsilon}^{\xi} \backslash F_{f, \varepsilon}^{\xi+1}$ for $\gamma, \xi<\alpha$. Assume that $|x-y|<\min (\delta(x), \delta(y))$ and suppose that $\gamma<\xi$. Since

$$
y \in(x-\delta(x), x+\delta(x)) \subset[0,1] \backslash F_{f, \varepsilon}^{\gamma+1},
$$

we get $y \notin F_{f, \varepsilon}^{\gamma+1}$, which contradicts $F_{f, \varepsilon}^{\xi} \subset F_{f, \varepsilon}^{\gamma+1}$. So $\gamma=\xi$.

Again from the definition of $\delta$, there exists $t \in F_{f, \varepsilon}^{\gamma} \backslash F_{f, \varepsilon}^{\gamma+1}$, such that $\delta^{t}(x)>\frac{3}{4} \delta(x)$. So $d\left(x,[0,1] \backslash V_{t}\right)>\frac{3}{2} \delta(x)>|x-y|$. Hence $x, y \in V_{t}$. From the definition of $V_{t}$ it follows that $|f(x)-f(y)|<\varepsilon$. $F_{f, \varepsilon}^{\gamma+1}$.

(3) The function $\delta$ restricted to the set $F_{f, \varepsilon}^{\gamma}$ is continuous on the set $F_{f, \varepsilon}^{\gamma} \backslash$

Proof. The function $\delta$ is defined on the set $F_{f, \varepsilon}^{\gamma} \backslash F_{f, \varepsilon}^{\gamma+1}$ as an upper bound of the family of functions that fullfill the Lipschitz condition with common constant 1. Moreover the set $F_{f, \varepsilon}^{\gamma} \backslash F_{f, \varepsilon}^{\gamma+1}$ is open with respect to $F_{f, \varepsilon}^{\gamma}$.

(4) $\beta(\delta) \leq \alpha$. 
Proof. For a given $\eta>0$ let us consider the sequence of sets $\left\{F_{\delta, \eta}^{\gamma}\right\}_{\gamma<\omega_{1}}$. We shall show by the transfinite induction that for every $\gamma<\omega_{1}$ we have

$$
F_{\delta, \eta}^{\gamma} \subset F_{f, \varepsilon}^{\gamma} .
$$

The inclusion is obvious for $\gamma=0$ because $[0,1] \subset[0,1]$. Suppose that for $\gamma<\omega_{1}$ the above inclusion holds. From (3) it follows that the oscillation of the function $\delta$ is equal to 0 in each point of the set $F_{f, \varepsilon}^{\gamma} \backslash F_{f, \varepsilon}^{\gamma+1}$ Hence $F_{\delta, \eta}^{\gamma+1} \subset F_{f, \varepsilon}^{\gamma+1}$. So $\beta(\delta, \eta) \leq \beta(f, \varepsilon) \leq \alpha$, and, as the number $\eta$ is arbitrary, we have $\beta(\delta) \leq \alpha$.

(5) The function $\delta$ is upper semicontinuous.

Consider $x_{0} \in[0,1]$ and a sequence $\left(x_{n}\right)_{n \in \mathbf{N}}$ convergent to $x_{0}$ such that $\lim _{n \rightarrow \infty} \delta\left(x_{n}\right)=g$. We shall show that $g \leq \delta\left(x_{0}\right)$. There exists the ordinal $\gamma<\alpha$, such that $x_{0} \in F_{f, \varepsilon}^{\gamma} \backslash F_{f, \varepsilon}^{\gamma+1}$. As $x_{0} \notin F_{f, \varepsilon}^{\gamma+1}$ and the set $F_{f, \varepsilon}^{\gamma+1}$ is closed we can assume that no term of the sequence $\left(x_{n}\right)$ belongs to $F_{f, \varepsilon}^{\gamma+1}$. There are two possibilities:

$1^{\circ}$ almost every term of the sequence $\left(x_{n}\right)$ belongs to $F_{f, \varepsilon}^{\gamma} \backslash F_{f, \varepsilon}^{\gamma+1}$;

$2^{\circ}$ infinitely many terms of that sequence belongs to $[0,1] \backslash F_{f, \varepsilon}^{\gamma}$.

In the first case we have $g=\lim _{n \rightarrow \infty} \delta\left(x_{n}\right)=\delta\left(x_{0}\right)$ as the function $\delta$ is continuous on $F_{f, \varepsilon}^{\gamma} \backslash F_{f, \varepsilon}^{\gamma+1}$.

In the second case we can assume that all terms of $\left(x_{n}\right)$ belong to $[0,1] \backslash F_{f, \varepsilon}^{\gamma}$. For a given $n$ in virtue of (1) we obtain

$$
x_{0} \notin\left(x_{n}-\delta\left(x_{n}\right), x_{n}+\delta\left(x_{n}\right)\right),
$$

hence

$$
\left|x_{0}-x_{n}\right| \geq \delta\left(x_{n}\right)>0
$$

and

$$
g=\lim _{n \rightarrow \infty} \delta\left(x_{n}\right)=0<\delta\left(x_{0}\right) .
$$

As a result $\delta$ is upper semicontinuous.

(6) The function $\delta$ belongs to $B 1^{*}$.

Let $F \subset[0,1]$ be an arbitrary nonempty closed set. We shall show existence of the interval $(a, b)$ such that $(a, b) \cap F \neq \emptyset$ and $\left.\delta\right|_{F}$ is continuous on $(a, b) \cap F$. Let $\gamma$ be the least ordinal such that $F \subset F_{f, \varepsilon}^{\gamma}$ but $F \not \subset F_{f, \varepsilon}^{\gamma+1}$. Let $x_{0} \in F \cap\left(F_{f, \varepsilon}^{\gamma} \backslash F_{f, \varepsilon}^{\gamma+1}\right)$. From (1) and (3), the function $\delta$ is continuous on the set $\left(x_{0}-\delta\left(x_{0}\right), x_{0}+\delta\left(x_{0}\right)\right) \cap F_{f, \varepsilon}^{\gamma}$, and hence continuous on the set $\left(x_{0}-\delta\left(x_{0}\right), x_{0}+\delta\left(x_{0}\right)\right) \cap F$. As a consequence $\delta \in B 1^{*}$. 
Remark 1 The last result is similar (but not comparable) to the main result of Atok, Tang and Zhao ([4], Theorem 2). Nevertheless we decided to demonstrate our theorem because the proof is completely different.

\section{References}

[1] A. Bruckner, Differentiation of Real Functions, CRM Monogr. Ser., 5 (1994).

[2] A. S. Kechris and A. Louveau, A classification of Baire class 1 functions, Trans. Amer. Math. Soc., 318(1) (1990), 209-236.

[3] P. Y.-Lee, W. Tang, and D. Zhao, An equivalent definition of functions of the first Baire class, Proc. Amer. Math. Soc., 129(8) (2000), 22732275 .

[4] Z. Atok, W.-K. Tang, and D. Zhao, Gauges of Baire class one functions, J. Math. Anal. Appl., 343(2) (2008), 866-870 